\title{
Hombres violentos contra la pareja: ¿tienen un trastorno mental y requieren tratamiento psicológico?
}

\section{Male batterers: are they mentally ill and are they needed of psychological treatment?}

\author{
Enrique Echeburúa \\ Universidad del País Vasco (UPV/EHU), España \\ Pedro Javier Amor \\ Universidad Nacional de Educación a Distancia (UNED), España
}

Rec (17 diciembre 2015) Acept (4 marzo 2016)

\begin{abstract}
Resumen
Hay muchas razones por las que los hombres maltratadores contra la pareja deben recibir tratamiento psicológico. En este artículo se analizan los transtornos más relevantes, tales como el abuso de alcohol/drogas, los celos patológicos y los transtornos de personalidad (antisocial, límite, narcisista y paranoide), así como los déficits psicológicos de estas personas, tales como el descontrol de la ira, las dificultades emocionales, las distorsiones cognitivas, la baja autoestima y los déficits de comunicación y de solución de problemas. Se describen las tipologías de hombres maltratadores más relevantes y se señala su importancia para la planificación del tratamiento individualizado. La inclusión de medidas penales y de tratamiento psicológico es posible. Se analiza la motivación para el tratamiento y se señalan las principales vías de intervención terapéutica, así como los resultados obtenidos. Por último, se comentan las líneas de investigación más urgentes para el futuro.
\end{abstract}

Palabras clave: violencia contra la pareja, maltratadores, transtornos mentales, tratamiento psicológico.

\begin{abstract}
There are many reasons why men who batter should be psychologically treated. In this paper the most relevant mental disorders, such as substance use disorder, pathological jealousy, and antisocial, borderline, narcissistic and paranoid personality disorders, and psychological deficits of batterer men, such as anger, emotional difficulties, cognitive distortions, low self-esteem and deficits in social skills and problem solving, are analyzed. Male domestic violence offender typologies according to the most relevant classifications are commented. The role of these typologies for treatment is pointed out. An analysis of how to combine court intervention and psychological treatment to rehabilitate abusive men is also carried out. Motivational enhancement strategies and effective therapy for men who batter are discussed. Finally, the future perspectives and the most relevant goals of research are commented on.

Key words: intimate partner violence, batterer men, mental disorders, psychological treatment.
\end{abstract}




\section{Introducción}

Las relaciones de pareja, libremente establecidas en la actualidad, están basadas en el intercambio de conductas gratificantes, lo que conlleva la posibilidad de su disolución si el balance de la relación es insatisfactorio. Por ello, la violencia contra la pareja puede considerarse algo anómalo. Cuando un hombre se implica en una relación violenta con una mujer con la que comparte o ha compartido voluntariamente sentimientos de intimidad y un proyecto de vida y con la que frecuentemente tiene hijos en común, cabe pensar que padece algún tipo de transtorno mental o de alteración psicológica (Brasfield, 2014).

No es fácil responder a la pregunta de por qué los hombres se comportan de forma violenta precisamente en la relación de pareja, que suele constituir un reducto de intimidad y de ternura. La conducta violenta en este contexto suele ser resultado de un estado emocional intenso -la ira-, que interactúa con unas actitudes previas de hostilidad, un repertorio de conductas pobres (déficits de habilidades de comunicación y de solución de problemas o dependencia emocional) y unos factores precipitantes, entre otros, las situaciones de estrés, el abuso de alcohol/drogas o los celos (Kelley, Edwards, Dardis, y Gidycz, 2015).

Hay ciertas circunstancias específicas de la relación de pareja que posibilitan esta secuencia crónica de conductas violentas. Un hombre tiende a descargar su ira específicamente en aquella persona que percibe como más vulnerable (una mujer) y en un entorno (la casa) en que es más fácil ocultar lo ocurrido. Además, los logros obtenidos con las conductas violentas previas desempeñan un papel muy importante. Muy frecuentemente el hombre maltratador ha conseguido los objetivos deseados con los comportamientos agresivos anteriores. Es decir, la violencia puede ser un método sumamente efectivo y rápido para salirse con la suya. A su vez, la sumisión de la mujer puede quedar también consolidada porque, con un comportamiento sumiso, consigue evitar las consecuencias negativas derivadas de una conducta violenta por parte de la pareja. Además las víctimas pueden sentirse incapaces de escapar del control de los agresores al estar sujetas a ellos por el miedo, la dependencia emocional, el aislamiento social o distintos tipos de vínculos económicos, legales o sociales (Jouriles y McDonald, 2015).

Si bien los transtornos mentales en sentido estricto son relativamente poco frecuentes en los maltratadores (cerca del $20 \%$ del total), los síntomas psicopatológicos son muy habituales, así como las alteraciones psicológicas en el ámbito del control de la ira, de la empatía y expresión de emociones, de las cogniciones sobre la mujer y la relación de pareja y de las habilidades de comunicación y de solución de problemas (Echeburúa, Fernández-Montalvo, y Amor, 2003).

El principal objetivo de este artículo es analizar los transtornos mentales, las alteraciones de la personalidad y los déficits psicológicos que están más frecuentemente presentes en los hombres implicados en relaciones violentas contra la pareja, así como plantear los retos de futuro más importantes para su tratamiento.

\section{Transtornos mentales}

Se ha encontrado una cierta relación entre la violencia contra la pareja y los transtornos mentales. Los más frecuentes son los transtornos psicóticos, en función de las ideas delirantes de celos o de persecución, y el abuso de alcohol y drogas, que pueden activar las conductas violentas en las personas impulsivas y descontroladas (Shorey, Fabres, Brasfield, y Stuart, 2012)

Lo más característico en los agresores es que tengan una historia psiquiátrica anterior (alrededor del $45 \%$ ) muy por encima de la tasa de prevalencia en la población general, que sería en torno al 15\%-20\% (Echeburúa et al., 2003). Con frecuencia los motivos de consulta más frecuentes suelen ser el abuso de alcohol, los transtornos emocionales (ansiedad y depresión) y los celos patológicos. Ahora bien, más que cuadros clínicos bien delimitados lo que suelen tener son múltiples síntomas psicopatológicos. Aun así, este hecho no permite establecer relaciones de causalidad o unidireccionalidad entre los síntomas o transtornos psicopatológicos presentes y la violencia contra la pareja.

Por último, los factores predictores más habituales de comportamientos violentos entre las personas con un transtorno mental son los siguientes: a) una historia previa de violencia o de victimización, con una personalidad premórbida anómala; b) la falta de conciencia de enfermedad y el consiguiente rechazo o abandono del tratamiento; c) los transtornos del pensamiento (ideas delirantes de amenaza/control, de celos o de identificación errónea) o de la percepción (alucinaciones que implican fuerzas externas controladoras del comportamiento), con pérdida del sentido de la realidad; d) el abuso de alcohol o drogas; y e) el aislamiento familiar y social, resultado de la estigmatización o de la discriminación (Caetano, Vaeth, y Ramisetty-Milker, 2008; Shiina, 2015).

\section{Consumo abusivo de alcohol y de drogas}

La agresión bajo la influencia directa del alcohol es muy variable y puede oscilar entre el $40 \%$ y el $90 \%$ de los casos (Stuart, 2005). Aunque el alcohol no explica en su totalidad 
las conductas violentas en los maltratadores, es el peor aliado de la violencia por múltiples aspectos (Catalá-Miñana, Lila, y Oliver, 2013; Echeburúa, Amor, y Fernández-Montalvo, 2002; Fals-Stewart y Kennedy, 2005; Stuart, 2005; Thompson y Kingree, 2006): a) actúa como facilitador y desinhibidor del comportamiento violento, que generalmente deriva de actitudes hostiles previas; b) sigue la ley dosis-efecto, según la cual cuanto mayor es el consumo mayor es la gravedad de la violencia ejercida contra la pareja; c) incrementa el riesgo de reincidencia en comportamientos violentos; y d) está relacionado con un peor pronóstico terapéutico.

Por lo que se refiere al consumo de drogas, las tasas de incidencia en los hombres violentos son menores y oscilan entre el $13 \%$ y el $35 \%$ de las personas estudiadas, pero tienden a aumentar entre los agresores más jóvenes (Slep, Foran, Heyman, Snarr, y USAF Family Advocacy Research Program, 2015).

\section{Celos patológicos}

Los celos patológicos se caracterizan por una preocupación excesiva e irracional sobre la infidelidad de la pareja, que provoca una intensa alteración emocional y que lleva a la persona a realizar conductas comprobatorias para controlar a su pareja. Lo que define la patología de los celos es la ausencia de una causa real desencadenante, la intensidad desproporcionada de los celos, el alto grado de interferencia con la vida cotidiana, el gran sufrimiento experimentado $\mathrm{y}$, en último término, la pérdida de control, con reacciones irracionales (Rodríguez, DiBello, y Neighbors, 2015).

Habitualmente, los celos patológicos suelen ser de tipo pasional o delirante. Cuando existen celos pasionales la persona sufre porque teme con mucha intensidad perder a su pareja y siente envidia de que esta pueda ser disfrutada por otro. En cambio, los celos delirantes parten de la presencia de una idea falsa objetivamente pero de la que la persona tiene una certeza absoluta de ser engañado. Este tipo de celos suele ser frecuente en los transtornos psicóticos (paranoia o esquizofrenia paranoide), así como en el alcoholismo.

Por otra parte, el riesgo de violencia vinculado a los celos en un caso y otro es distinto. En los celos pasionales se ve afectada la autoestima de la persona y experimenta una elevada ansiedad que se puede cargar de agresividad y de obcecación hasta acabar en comportamientos violentos. Asimismo, las conductas violentas graves se cometen bajo los efectos de una gran tensión emocional, que enturbia la conciencia, cuando existen conductas controladoras extremas y sentimientos de posesión. Así, la violencia se dispara cuando el sujeto experimenta un ataque de celos y se siente despechado, sobre todo si ha mostrado comportamientos agresivos previos, carece de una autoestima y de unas habilidades sociales adecuadas y cuenta con un repertorio de conductas y de intereses muy limitado. De forma diferente, el riesgo de agresión a la mujer en el caso de los celos delirantes es muy alto cuando los celos se han consolidado y existe un claro deterioro de la relación de pareja.

Estos tipos de celos, caracterizados por ser infundados o desproporcionados, no tienen nada que ver con los celos normales que se refieren a la relación de compromiso y de angustia emocional cuando uno piensa que la pareja pudiera implicarse en una relación sentimental con otra persona (Rodríguez et al., 2015).

\section{Transtornos de personalidad}

Se han identificado diferentes transtornos de la personalidad relacionados con las conductas violentas contra la pareja (Amor, Echeburúa, y Loinaz, 2009; Esbec y Echeburúa, 2010; Lila, Gracia, y Herrero, 2012). Generalmente los que están más relacionados con los comportamientos violentos más graves son aquellos pertenecientes al grupo $\mathrm{B}$ de los transtornos de la personalidad y que se caracterizan por la inmadurez, la emotividad y la inestabilidad. En concreto, el transtorno antisocial de la personalidad (la psicopatía), caracterizado por la manipulación, por la falta de empatía en las relaciones interpersonales y por la ausencia de remordimiento ante el dolor causado, propicia la aparición de conductas violentas y crueles. Cuando el maltratador es un psicópata, habitualmente plantea exigencias irracionales, muestra un desapego hacia los hijos, abusa del alcohol o de las drogas, no tiene amigos y es un manipulador que utiliza a los demás en su beneficio (Echeburúa y FernándezMontalvo, 2007).

El transtorno de personalidad narcisista -aunque comparte algunas características con el transtorno anterior, como son la manipulación e instrumentalización de los demás y la falta de empatía- se caracteriza fundamentalmente por tener sentimientos de grandeza y prepotencia junto con una gran necesidad de estimación permanente.

A su vez, el transtorno límite o borderline, en el que son frecuentes la impulsividad, el miedo al abandono, la inestabilidad emocional y un sentimiento crónico de vacío, facilita la aparición de conductas impredecibles en la relación de pareja (Anita, O’Leary, Graña, y Foran, 2014; Huss y Langhinrichsen-Rohling, 2006).

Por último, el transtorno de personalidad paranoide (perteneciente al grupo A), en el que la desconfianza, los celos (en este caso) y los recelos están presentes de forma 
constante, también pueden dar lugar a comportamientos violentos contra la pareja.

En resumen, hay cuatro dimensiones de personalidad implicadas generalmente en las conductas violentas: 1) la impulsividad; 2) la falta de regulación emocional; 3) el narcisismo y las amenazas al yo; y 4) el estilo de personalidad paranoide. Las dos últimas están específicamente relacionadas con la violencia y los transtornos mentales (Echauri, Fernández-Montalvo, Martínez, y Azcárate, 2011).

\section{Alteraciones psicológicas}

Las alteraciones psicológicas pueden ser muy variables, pero, de una forma u otra, y a diferencia de los transtornos mentales, están presentes en todos los casos. Entre ellas pueden darse la falta de control sobre la ira, las dificultades en la expresión de emociones, las distorsiones cognitivas, el déficit de habilidades de comunicación y de solución de problemas y la baja autoestima (Loinaz, Echeburúa, y Ullate, 2012).

\section{Falta de control sobre la ira}

Los hombres violentos contra la pareja presentan niveles moderadamente superiores de ira y hostilidad que aquellos que no lo son (Norlander y Eckhardt, 2005). A su vez, gran parte de este tipo de agresores se caracterizan por la impulsividad, la pérdida de control sobre la ira y por actitudes de hostilidad hacia la pareja. Sin embargo, no todos ellos tienen problemas con el control de la ira. Murphy, Taft, y Eckhardt (2007) identificaron tres tipos de agresores, de los cuales el grupo de ira normal no tenía problemas para controlar esta emoción, se comportaba con una violencia menos grave y mostraba un mejor pronóstico terapéutico que los restantes grupos -ira patológica y bajo control de la ira-. En definitiva, los que ejercen una violencia más grave presentan niveles más elevados de ira y de hostilidad (Norlander y Eckhardt, 2005), así como un peor pronóstico.

En muchos casos la ira es la respuesta a una situación de malestar en la convivencia o una forma inadecuada de hacer frente a los problemas cotidianos (por ejemplo, a las dificultades en la educación de los hijos o a la falta de acuerdos sobre el ocio, el control de la economía o los espacios de intimidad personal) (Eckhardt, Samper, y Murphy, 2008).

\section{Dificultades en la expresión y captación de emociones}

Muchos conflictos en las relaciones de pareja tienen que ver con las dificultades en la expresión y en la captación de emociones. En este sentido, muchos agresores tienen dificultades para expresar sus sentimientos o no han aprendido a expresarlos adecuadamente; también es posible que muchos de ellos no sepan captar o interpretar adecuadamente los sentimientos de su pareja.

A su vez, la inhibición de los sentimientos y una percepción distorsionada de la realidad (por ejemplo, percibir situaciones como amenazantes cuando no lo son) pueden llevar a conflictos que, al no saber resolverse de otra manera, se expresan de forma violenta. En definitiva, este analfabetismo emocional dificulta el establecimiento de relaciones de intimidad o de amistad profunda y facilita los comportamientos violentos cuando se dan otros factores (por ejemplo, hostilidad contra la pareja, distorsiones cognitivas, ira, estrés, etc.).

\section{Distorsiones cognitivas sobre la mujer y la relación de pareja}

Los hombres maltratadores suelen estar afectados de partida por numerosos sesgos cognitivos, que implican creencias equivocadas sobre los roles de género y sobre la supuesta inferioridad de la mujer con respecto al hombre, así como ideas distorsionadas sobre la legitimación de la violencia como forma de resolver los conflictos.

A su vez, dentro del contexto de la violencia de pareja, muchos agresores emplean estrategias de afrontamiento para eludir la responsabilidad propia de las conductas violentas mediante su negación, minimización o justificación (Dutton, 2007). Pueden alegar que se trata de un problema que afecta a ambos ( "en todas las parejas hay problemas") o que es por culpa de la mujer ("fue ella la que me provocó; es ella la que tiene que cambiar"), hacer atribuciones externas ("los problemas del trabajo me hacen perder el control") o incluso personales ("estoy pasando una mala racha”) que de algún modo eluden la responsabilidad de los comportamientos violentos o restan importancia a las consecuencias negativas de esa conducta para la víctima (Echeburúa y Fernández-Montalvo, 2009).

Este tipo de distorsiones cognitivas tienen un claro paralelismo con los mecanismos de desconexión moral planteados por Bandura (2002), que, en el caso que nos ocupa, posibilitarían que el agresor no sufriera emocionalmente en exceso ante la violencia ejercida contra su pareja. Algunos de estos mecanismos tienen que ver con la justificación moral del maltrato, la minimización, la negación o el falseamiento de las consecuencias de la violencia ejercida, el desplazamiento o la difusión de la responsabilidad y la deshumanización o atribución de culpa a la propia víctima. En definitiva, 
cuando una conducta genera malestar al pensar fríamente en ella o es rechazada socialmente, se utilizan estrategias de afrontamiento o distorsiones cognitivas para eludir la responsabilidad y atenuar el malestar interno que podría generar en el agresor.

\section{Déficits en habilidades de comunicación y de solución de problemas}

Los maltratadores suelen presentar unas habilidades de comunicación muy pobres y una baja tolerancia a la frustración, así como estrategias inadecuadas para solucionar los problemas (Kelley et al., 2015). Estos déficits, unidos a los conflictos y los problemas cotidianos, pueden generar un estrés permanente y actuar como desencadenantes de los episodios violentos contra la pareja.

\section{Baja autoestima}

Muchos agresores que se caracterizan por tener baja autoestima tratan de dominar y controlar obsesivamente a su pareja o incluso pueden recurrir a la violencia como medio para conseguir una estima que no logran de otra forma. Sin embargo, la reiteración de la violencia no hace sino empeorar la baja autoestima del agresor (Donahue, McClure, y Moon, 2014). En estos casos se podría hablar de una violencia por compensación en donde quien ejerce la violencia muestra una gran inseguridad e intenta superar sus frustraciones con quien tiene más a mano y no le va a responder (Echeburúa et al., 2003). A su vez, los agresores que tienen baja autoestima tienden a percibir muchas situaciones como amenazantes y a minimizar la gravedad de su comportamiento violento (Lila et al., 2012), algo que es de gran relevancia dentro del contexto de la intervención psicológica.

Sin embargo, en otros casos los agresores se perciben o se muestran ante los demás con una elevada autoestima que, lejos de ser saludable, no es más que el reflejo de una distorsión de su autoimagen con tintes narcisistas que igualmente denota una profunda inseguridad. Estas personas pueden vulnerar los derechos de los demás, mostrar poca empatía y comportarse violentamente ante situaciones percibidas como una afrenta a su dignidad (llevarles la contraria, quitarles autoridad ante otras personas, etc.).

\section{Tipos de maltratadores}

Los hombres violentos contra la pareja no responden a un perfil homogéneo (Amor et al., 2009; Dixon y Browne, 2003) y, desde esta perspectiva, parece oportuno diseñar y aplicar estrategias de intervención ajustadas a cada tipología.
A pesar de determinados matices diferenciales de unos estudios a otros, existen tres tipos (Tabla 1) -sobrecontrolados, disfóricos/borderline y antisociales/violentos en general-, que se diferencian en cuanto a la mayor o menor presencia de determinados transtornos psicopatológicos (generalmente los de la personalidad), a la extensión, frecuencia y gravedad de la violencia ejercida, así como al riesgo que representan para la víctima (Amor et al., 2009; Cavanaugh y Gelles, 2005; Holtzworth-Munroe y Stuart, 1994).

Es más, estos tres tipos de agresores también se han identificado cuando se analizan las tipologías de hombres que han asesinado a su pareja (Kivisto, 2015). En estos casos, habitualmente los disparadores del homicidio pueden

Tabla 1. Paralelismos entre tipologías de hombres violentos contra la pareja (Amor et al., 2009; Cavanaugh y Gelles, 2005; Holtzworth-Munroe y Stuart; 1994; Holtzworth-Munroe et al., 2000; Monson y Langhinrichsen-Rohling, 1998)

\begin{tabular}{|c|c|c|c|}
\hline & Sobrecontrolados & $\begin{array}{l}\text { Disfóricos/ } \\
\text { Borderline }\end{array}$ & Antisociales \\
\hline $\begin{array}{l}\text { Prevalencia } \\
\text { estimada }\end{array}$ & $36 \%-50 \%$ & $15 \%-25 \%$ & $16 \%-25 \%$ \\
\hline $\begin{array}{l}\text { Riesgo } \\
\text { potencial de } \\
\text { violencia } \\
\text { grave }\end{array}$ & Bajo & Medio & Alto \\
\hline $\begin{array}{l}\text { Antecedentes } \\
\text { penales }\end{array}$ & No & $\begin{array}{c}\text { Habitualmente } \\
\text { no }\end{array}$ & Sí \\
\hline \multirow[t]{2}{*}{$\begin{array}{c}\text { Características } \\
\text { de la } \\
\text { violencia }\end{array}$} & $\begin{array}{l}\text { - Baja } \\
\text { frecuencia y } \\
\text { gravedad }\end{array}$ & $\begin{array}{c}\text { - Moderada } \\
\text { frecuencia y } \\
\text { gravedad }\end{array}$ & $\begin{array}{c}\text {-Alta } \\
\text { frecuencia y } \\
\text { gravedad }\end{array}$ \\
\hline & $\begin{array}{l}\text {-Limitada al } \\
\text { hogar }\end{array}$ & & -Generalizada \\
\hline $\begin{array}{c}\text { Psicopatología } \\
\text { del eje I }\end{array}$ & No es frecuente & $\begin{array}{c}\text { Sí } \\
\text { (sintomatología } \\
\text { depresiva) }\end{array}$ & $\begin{array}{l}\text { Sí (abuso } \\
\text { de alcohol } \\
\text { y de otras } \\
\text { sustancias) }\end{array}$ \\
\hline \multirow[t]{3}{*}{$\begin{array}{c}\text { Psicopatología } \\
\text { del eje II }\end{array}$} & $\begin{array}{l}\text { - Narcisismo } \\
\text { subclínico }\end{array}$ & $\begin{array}{l}\text { - Trastorno } \\
\text { borderline }\end{array}$ & $\begin{array}{c}\text { - Trastorno } \\
\text { antisocial } \\
\text { y alta } \\
\text { psicopatía }\end{array}$ \\
\hline & $\begin{array}{l}\text { - Posibles rasgos } \\
\text { compulsivos, } \\
\text { histriónicos, } \\
\text { esquizoides, de } \\
\text { dependencia }\end{array}$ & & $\begin{array}{l}\text { - Trastorno } \\
\text { narcisista }\end{array}$ \\
\hline & $\begin{array}{l}\text { - Tendencia } \\
\text { pasivo-agresiva }\end{array}$ & & \\
\hline
\end{tabular}

Nota. En el estudio empírico de Holtzworth-Munroe et al. (2000) se identificó una cuarta tipología denominada "Antisocial de bajo nivel" (33\% de los casos). 
estar relacionados fundamentalmente con el abandono en la tipología antisocial, con el miedo al abandono y los celos en el grupo disfórico/borderline o, por contraposición, con el miedo a sentirse atrapados en la relación y el padecimiento de crisis catatímicas (actos repentinos y aislados que derivan de un estado de tensión con una fuerte carga emocional) en el caso de los agresores sobrecontrolados. Según Kivisto (2015), existe una cuarta tipología de "enfermos mentales", que se caracterizan por tener un transtorno psicótico o una depresión grave en el momento del homicidio que no guarda relación directa con el abandono o los celos.

Por otra parte, los tipos de agresores contra la pareja se pueden establecer en función de dos dimensiones: la extensión de la violencia y el perfil psicopatológico presentado (Fernández-Montalvo y Echeburúa, 1997).

Por lo que se refiere a la extensión de la violencia, la mayor parte de los sujetos (el 74\%) son violentos exclusivamente contra la pareja. Se trata de personas que con su pareja ejercen un nivel de maltrato grave, pero que en el resto de las relaciones interpersonales adoptan conductas adecuadas. En estos casos las frustraciones cotidianas, así como el abuso de alcohol o los celos patológicos, contribuyen a desencadenar episodios de violencia contra la pareja.

Por el contrario, los violentos generalizados (el 26\%), en los que son frecuentes las experiencias de maltrato en la infancia, son personas agresivas tanto con su pareja como con las demás personas y cuentan con muchas ideas distorsionadas sobre la utilización de la violencia como forma aceptable de arreglar los conflictos.

En cuanto al perfil psicopatológico, hay agresores con déficits en las habilidades interpersonales (el 55\%), es decir, que no han aprendido unas habilidades adecuadas para las relaciones interpersonales debido a carencias habidas en el proceso de socialización. El recurso a la violencia suple la ausencia de otro tipo de estrategias de solución de problemas.

Por otra parte, los maltratadores sin control de los impulsos (el $45 \%$ ) son personas que tienen episodios bruscos e inesperados de descontrol con la ira. Si bien presentan unas habilidades sociales más adecuadas y son más conscientes de que la violencia no es una forma aceptable de resolver los conflictos, se muestran incapaces de controlar los episodios violentos, que surgen en forma de un transtorno explosivo intermitente.

\section{¿Por qué hay que tratar psicológicamente a los hombres violentos contra la pareja?}

El tratamiento psicológico a los maltratadores es, junto con otras actuaciones judiciales y sociales, una medida útil, necesaria y posible. Un tratamiento psicológico puede ser de utilidad para que los agresores aprendan a afrontar sus limitaciones al no contar con las habilidades necesarias para resolver los problemas de pareja en la vida cotidiana. También puede servir para que haya un mayor control del agresor y para prevenir futuros episodios de violencia con la pareja o expareja y otras personas del entorno familiar. Es más, incluso el fracaso de la intervención con el hombre puede ayudar a la mujer en su proceso de toma de decisiones (por ejemplo, que ella decida abandonar definitivamente la relación de pareja). En la Tabla 2 se indican algunas de las razones por las que resulta necesaria la intervención psicológica con hombres violentos en el hogar.

Tabla 2. Razones de la intervención psicológica con hombres violentos contra la pareja

\section{Separación de la pareja}

- Riesgo de homicidio contra la expareja.

- Acoso, hostigamiento y reiteración de la violencia.

- Relación obligada de la mujer con el agresor por diversos vínculos (familiares, económicos, sociales, etc.), que la pone en riesgo de ser revictimizada.

- Posibilidad de reincidencia de los malos tratos con los hijos/as.

- Posibilidad de reincidencia de los malos tratos con otra mujer.

\section{Convivencia con la pareja}

- Peligro de que el maltrato continúe o de que, si cesó anteriormente, aparezca de nuevo.

- Alta probabilidad de que la violencia se extienda a otros miembros de la familia (hijos, personas mayores, etc.).

- Malestar psicológico del agresor, de la víctima y del resto de las personas que conviven en el hogar.

Por otra parte, tratar psicológicamente a un maltratador es hoy posible, sobre todo si la persona asume la responsabilidad de sus conductas y cuenta con una mínima motivación para el cambio. La dificultad mayor radica en su falta de motivación para reconocer el problema y tomar la decisión de cambiar. De hecho, muchos hombres acuden a tratamiento por mandato judicial, algo que se contrapone a una motivación auténtica para el cambio. Generalmente, la decisión genuina de acudir a un programa terapéutico se adopta solo cuando se dan varios requisitos previos en el individuo: reconocer que existe un problema; darse cuenta de que no lo puede resolver por sí solo; y, por último, valorar que el posible cambio va a mejorar su nivel de bienestar. Una guía básica de tratamiento figura descrita en la Tabla 3. 
Y REQUIEREN TRATAMIENTO PSICOLÓGICO?

Tabla 3. Guía básica de tratamiento y prevención de la reincidencia (Adaptación de Echeburúa et al., 2009)

\section{Guía básica de tratamiento}

Aspectos psicopatológicos y técnicas terapéuticas

- Déficit de empatía y analfabetismo emocional.

- Ejercicios para desarrollar la empatía (vídeos, relatos autobiográficos, testimonios, etc.) y técnicas de expresión de emociones.

- Ideas distorsionadas sobre la mujer, los roles sexuales y la violencia.

- Educación sobre la igualdad de los sexos.

- Reestructuración cognitiva.

- Ira descontrolada.

- Psicoeducación (ciclo de la violencia y proceso de escalada de la ira)

- Suspensión temporal.

- Distracción cognitiva.

- Entrenamiento en autoinstrucciones.

- Problemas con el control de emociones: ansiedad/estrés, sintomatología depresiva (tristeza), celos patológicos.

- Reestructuración cognitiva.

- Relajación.

- Actividades gratificantes.

- Déficits de asertividad y de comunicación

- Entrenamiento en asertividad y habilidades de comunicación.

- Déficits en la resolución de problemas

- Entrenamiento en solución de problemas.

- Déficits en las relaciones sexuales

- Educación sobre la sexualidad en la pareja.

\section{Guía de prevención de la reincidencia}

Aspectos psicopatológicos y técnicas terapéuticas

- Déficits en autoestima

- Reevaluación cognitiva.

- Establecimiento de metas positivas.

- Problemas con el consumo abusivo de alcohol y drogas

- Programa de consumo controlado.

- Riesgo de reincidencia

- Identificación de situaciones de riesgo.

- Aprendizaje de estrategias de afrontamiento.

- Creación de un estilo de vida positivo.
Desde esta perspectiva, el hombre violento va a estar realmente motivado cuando llega a percatarse de que los inconvenientes de seguir maltratando a su pareja superan a las ventajas de hacerlo. El terapeuta debe ayudar al agresor a lograr esa atribución correcta de la situación actual y a descubrirle las soluciones a su alcance (Carbajosa, Boira, y Tomás-Aragonés, 2013).

Ahora bien, existe mucha variabilidad de unos casos a otros con respecto a la motivación para el cambio y no es frecuente que desde el inicio del tratamiento se cumplan con los requisitos anteriormente señalados. Por esta razón es fundamental desde el comienzo de la intervención psicológica, y a lo largo de todo el proceso terapéutico, abordar la motivación para el cambio y la percepción que se tiene sobre el problema de la violencia.

Según Murphy y Eckhardt (2005), hay diversas estrategias motivacionales que se pueden diferenciar según la fase del tratamiento. Así, en las primeras fases convendría utilizar, entre otros aspectos, un alto nivel de empatía y de escucha reflexiva, clarificar los roles con respecto a la pareja y la fuente de derivación, dejar que la persona siga su propio proceso de cambio (sin forzarle a cambiar), prestar atención a signos potenciales de la motivación para el cambio y facilitarle verbalizaciones que vayan en esa dirección. En fases más avanzadas de la intervención se debería prestar atención a los signos de ambivalencia y ayudar a resolverlos mediante la reflexión precisa, generando afirmaciones automotivadoras, evitando conceptualizaciones y objetivos del tratamiento que estén centrados en los problemas de los demás, desarrollando un plan de cambio coherente con objetivos claros compartidos por la persona y centrándose en la recuperación a largo plazo y en el proceso de cambio (más que en la recuperación de la relación de pareja).

En definitiva, las técnicas motivacionales pueden disminuir la resistencia hacia la terapia y mejorar la implicación en el tratamiento, así como predecir buenos resultados terapéuticos. Si los agresores reciben este entrenamiento motivacional antes de comenzar propiamente con el tratamiento, se responsabilizan más de su comportamiento violento, culpan menos a los demás, tienen mejores expectativas respecto a la terapia y muestran una mayor adherencia al tratamiento (Echeburúa, Sarasua, Zubizarreta, y Corral, 2009).

Por último, se puede decir que aunque el nivel de rechazos y abandonos prematuros del tratamiento psicológico todavía es alto, muchos de los hombres que completan la intervención logran reducir las conductas de maltrato y suelen evitar la reincidencia, lo que contribuye a un mayor bienestar para el agresor y para la víctima (Austin y Dankwort, 1999; Babcock y Steiner, 1999). 


\section{Discusión}

Los hombres violentos contra la pareja no son habitualmente enfermos mentales, pero presentan déficits psicológicos importantes que son susceptibles de tratamiento. Una razón adicional para el tratamiento de los agresores es el carácter crónico de la violencia contra la pareja. Se trata de una conducta sobreaprendida por parte del maltratador, que está consolidada en su repertorio de conductas por los beneficios logrados: obtención de la sumisión de la mujer, sensación de poder, etc. Si se produce una separación o divorcio y el hombre violento se vuelve a emparejar, se puede predecir que va a haber más adelante una repetición de las conductas de maltrato con la nueva pareja. Por ello, la prevención de futuras víctimas también hace aconsejable el tratamiento psicológico del agresor (Arias, Arce, y Vilariño, 2013).

Ya hay programas de tratamiento disponibles para hombres violentos contra la pareja, bien en un régimen comunitario, bien en prisión o bajo control judicial. Tratar a un maltratador no significa verlo como enfermo y considerarle no responsable de sus conductas violentas. No se trata de estigmatizar a estas personas, sino de ayudarles a superar sus carencias psicológicas (Carbajosa et al., 2013; Echeburúa et al., 2009)

El tratamiento psicológico resulta un instrumento útil solo en aquellos casos en los que el agresor es consciente de su problema, asume su responsabilidad en los episodios de violencia y se muestra motivado para modificar su comportamiento agresivo. Solo con un enfoque amplio, en donde se integren medidas judiciales, sociales y psicológicas o médicas, se puede hacer frente de forma eficaz a la violencia contra la pareja. Los tratamientos psicológicos de hombres violentos en el hogar ofrecen unos resultados aceptables cuando los sujetos concluyen el programa propuesto, si bien el nivel de rechazos y abandonos prematuros es todavía alto (Echeburúa, Fernández-Montalvo, y Amor, 2006; Lila, Oliver, Galiana, y Gracia, 2013).

La intervención clínica puede llevarse a cabo en un entorno comunitario, especialmente cuando la pareja sigue unida y cuando la violencia no es excesivamente grave, o en un medio judicial, cuando el agresor está con la suspensión de la pena o recluido en prisión, especialmente cuando está próximo a la excarcelación. En este último caso, se trata de que el maltratador, al que se considera responsable de los delitos violentos cometidos contra su pareja, no reincida cuando recupere la vida en libertad (Novo, Fariña, Seijo, y Arce, 2012).

Algunas de las líneas de investigación más prometedoras para el futuro son las siguientes: a) examinar la respuesta de diferentes subtipos de hombres violentos a distintos programas de tratamiento; $b$ ) analizar la efectividad de diversos tratamientos según la fuente de derivación (voluntarios versus obligados judicialmente) y el contexto cultural; $\mathrm{y}$ c) evaluar la necesidad de intervenciones más amplias mediante programas de tratamiento multicomponentes para aquellos agresores con diversos problemas psicopatológicos (por ejemplo, dependencia al alcohol y drogas o transtornos de personalidad) (Murphy et al., 2007). En cualquier caso, la motivación para el tratamiento es el motor del cambio y la piedra angular del éxito en un programa terapéutico con maltratadores. No se trata tan solo de la motivación inicial para acudir a la consulta, sino de la motivación necesaria para mantenerse en el tratamiento y cumplir adecuadamente con las prescripciones terapéuticas (Echeburúa, Sarasua, Zubizarreta, Amor, y Corral, 2010).

\section{Referencias}

Amor, P. J., Echeburúa, E., y Loinaz, I. (2009). ¿Se puede establecer una clasificación tipológica de los hombres violentos contra su pareja? International Journal of Clinical and Health Psychology, 9, 519-539.

Anita, J., O’Leary, D., Graña, J. L., y Foran, H. M. (2014). Risk factors for men's intimate physical aggression in Spain. Journal of Family Violence, 29, 287-297.

Arias, E., Arce, R., y Vilariño, M. (2013). Batterer intervention programmes: A meta-analytic review of effectiveness. Psychosocial Intervention, 22, 153-160

Austin, J. B., y Dankwort, J. (1999). Standards for batterer programs: A review and analysis. Journal of Interpersonal Violence, 14, 152-168.

Babcock, J. C., y Steiner, R. (1999). The relationship between treatment, incarceration, and recidivism of battering: A program evaluation of Seattle's coordinated community response to domestic violence. Journal of Family Psychology, 13, 46-59.

Bandura, A. (2002). Selective moral disengagement in the exercise of moral agency. Journal of Moral Education, 31, 101-119.

Brasfield, R. (2014). The absence of evidence is not the evidence of absence: The abusive personality as a disordered mental state. Aggression and Violent Behavior, 19, 515-522.

Caetano, R., Vaeth, P., y Ramisetty-Mikler, S. (2008). Intimate Partner Violence Victim and Perpetrator Characteristics Among Couples in the United States. Journal of Family Violence, 23, 507-518.

Carbajosa, P., Boira, S., y Tomás-Aragonés, L. (2013). Difficulties, skills and therapy strategies in interventions with court-ordered batterers in Spain. Aggression and Violent Behavior, 18, 118-124.

Catalá-Miñana, A., Lila, M., y Oliver, A. (2013). Consumo de alcohol en hombres penados por violencia contra la pareja: Factores individuales y contextuales. Adicciones, 25, 19-28.

Cavanaugh, M. M., y Gelles, R. J. (2005). The Utility of Male Domestic Violence Offender Typologies: New Directions for Research, Policy, and Practice. Journal of Interpersonal Violence, 20, 155-166.

Dixon, L., y Browne, K. (2003). The heterogeneity of spouse abuse: A review. Aggression and Violent Behavior, 8, 107-130.

Donahue, J. J., McClure, K. S., y Moon, S. M. (2014). The relationship between emotion regulation difficulties and psychopathic personality characteristics. Personality Disorders: Theory, Research, and Treatment, 5, 186-194.

Dutton, D. G. (2007). The abusive personality. Violence and control in intimate relationships ( $2^{\mathrm{a}}$ ed.). New York: The Guilford Press. 
Echauri, J. A., Fernández-Montalvo, J., Martínez, M. A., y Azcárate, J. M. (2011). Transtornos de personalidad en hombres maltratadores a la pareja: perfil diferencial entre agresores en prisión y agresores con suspensión de condena. Anuario de Psicología Jurídica, 21, 97-105.

Echeburúa, E., Amor, P. J., y Fernández-Montalvo, J. (2002). Vivir sin violencia. Madrid. Pirámide.

Echeburúa, E., y Fernández-Montalvo, J. (2007). Male batterers with and without psychopathy: An exploratory study in Spanish prisons. International Journal of Offender Therapy and Comparative Criminology, 51, 254-263.

Echeburúa, E., y Fernández-Montalvo, J. (2009). Evaluación de un programa de tratamiento en prisión de hombres condenados por violencia grave contra la pareja. International Journal of Clinical and Health Psychology, 9, 5-20.

Echeburúa, E., Fernández-Montalvo, J., y Amor, P. J. (2003). Psychopathological profile of men convicted of gender violence: A study in the prisons of Spain. Journal of Interpersonal Violence, 18, 798-812.

Echeburúa, E., Fernández-Montalvo, J., y Amor, P. J. (2006). Psychological treatment of men convicted of gender violence: A pilot-study in Spanish prisons. International Journal of Offender Therapy and Comparative Criminology, 50, 57-70.

Echeburúa, E., Sarasua, B., Zubizarreta, I., Amor, P. J., y Corral, P. (2010). Variables predictoras del rechazo, abandono y fracaso terapéutico en hombres violentos contra su pareja tratados psicológicamente en un marco comunitario. International Journal of Clinical and Health Psychology, 10, 403-420.

Echeburúa, E., Sarasua, B., Zubizarreta, I., y Corral, P. (2009). Evaluación de la eficacia de un tratamiento cognitivo-conductual para hombres violentos contra la pareja en un marco comunitario: una experiencia de 10 años (1997-2007). International Journal of Clinical and Health Psychology, 9, 199-217.

Eckhardt, C. I., Samper, R. E., y Murphy, C. M. (2008). Anger disturbances among perpetrators of intimate partner violence: Clinical characteristics and outcomes of court-mandated treatment. Journal of Interpersonal Violence, 23, 1600-1617.

Esbec, E., y Echeburúa, E. (2010). Violencia y transtornos de la personalidad: implicaciones clínicas y forenses. Actas Españolas de Psiquiatría, $38,249-261$.

Fals-Stewart, W., y Kennedy, C. (2005). Addressing intimate partner violence in substance-abuse treatment. Journal of Substance Abuse Treatment, 29 5-17.

Fernández-Montalvo, J., y Echeburúa, E. (1997). Variables psicopatológicas y distorsiones cognitivas de los maltratadores en el hogar: un análisis descriptivo. Análisis y Modificación de Conducta, 23, 151-180.

Holtzworth-Munroe, A., Meehan, J. C., Herron, K., Rehman, U., y Stuart, G. L. (2000). Testing the Holtzworth-Munroe and Stuart (1994) batterer typology. Journal of Consulting and Clinical Psychology, 68, 1000-1019.

Holtzworth-Munroe, A., y Stuart, G. L. (1994). Typologies of male batterers: Three subtypes and the differences among them. Psychological Bulletin, 116, 476-497.

Huss, M. T., y Langhinrichsen-Rohling, J. (2006). Assessing generalization of psychopathy in a clinical sample of domestic violence perpetrators. Law and Human Behaviour, 30, 571-586.

Jouriles, E. N., y McDonald, R. (2015). Intimate partner violence, coercive control, and child adjustment problems. Journal of Interpersonal Violence, 30, 459-474.

Kelley, E. L, Edwards, K. M., Dardis, C. M., y Gidycz, C. A. (2015). Motives for physical dating violence among college students: A gendered analysis. Psychology of Violence, 5, 56-65.

Kivisto, A. J. (2015). Male perpetrators of intimate partner homicide: A review and proposed typology. Journal of the American Academy of Psychiatry and the Law, 43, 300-312.

Lila, M., Gracia, E., y Herrero, J. (2012). Asunción de responsabilidad en hombres maltratadores: Influencia de la autoestima, la personalidad narcisista y la personalidad antisocial. Revista Latinoamericana de Psicología, 44, 99-108.
Lila, M., Oliver, A., Galiana, L., y Gracia, E. (2013). Predicting success indicators of an intervention programme for convicted intimate-partner violence offenders: The Contexto Programme. The European Journal of Psychology Applied to Legal Context, 5, 73-95.

Loinaz, I., Echeburúa, E., y Ullate, M. (2012). Estilo de apego, empatía y autoestima en agresores de pareja. Terapia Psicológica, 30, 61-70.

Monson, C. M., y Langhinrichsen-Rohling, J. (2002). Sexual and nonsexual dating violence perpetration: Testing an integrated perpetrator typology. Violence and Victims, 17, 403-428.

Murphy, C. M., y Eckhardt, C. I. (2005). Treating the Abusive Partner. New York: The Guilford Press.

Murphy, C. M., Taft, C. T., y Eckhardt, C. I. (2007). Anger problem profiles among partner violent men: Differences in clinical presentation and treatment outcome. Journal of Counseling Psychology, 54, 189-200.

Norlander, B., y Eckhardt, C. (2005). Anger, hostility, and male perpetrators of intimate partner violence: A meta-analytic review. Clinical Psychology Review, 25, 119-152.

Novo, M., Fariña, F., Seijo, D., y Arce, R. (2012). Assessment of a community rehabilitation programme in convicted male intimate-partner violence offenders. International Journal of Clinical and Health Psychology, 12, 219-234.

Rodriguez, L. M., DiBello, A. M., y Neighbors, C. (2015). Positive and negative jealousy in the association between problem drinking and IPV perpetration. Journal of Family Violence, 30, 987-997.

Shiina, A. (2015). Risk Assessment and Management of Violence in Patients with Mental Disorders: A Review. Journal of Forensic, Legal y Investigative Sciences, 1, 1-5.

Shorey, R. C., Fabres, J., Brasfield, H., y Stuart, G. L. (2012). The prevalence of mental health problems in men arrested for domestic violence. Journal of Family Violence, 27, 741-748.

Slep, A. M. S., Foran, H. M., Heyman, R. E., Snarr, J. D., y USAF Family Advocacy Research Program (2015). Identifying unique and shared risk factors for physical intimate partner violence and clinically-significant physical intimate partner violence. Aggressive Behavior, 41, 227-241.

Stuart, G. L. (2005). Improving violence intervention outcomes by integrating alcohol treatment. Journal of Interpersonal Violence, 20, 388-393.

Thompson, M. P., y Kingree, J. B. (2006). The roles of victim and perpetrator alcohol use in intimate partner violence outcomes. Journal of Interpersonal Violence, 21, 163-177. 
